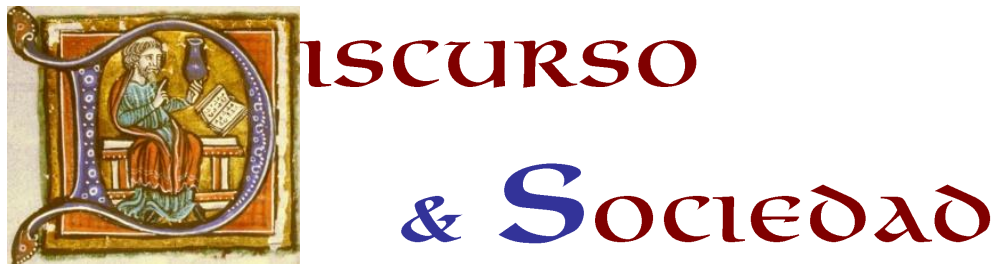

Copyright @ 2017

ISSN 1887-4606

Vol. 11(4), 537-570

www.dissoc.org

Artículo

\title{
La orientación discursiva de la universidad pública colombiana en el contexto de la globalización neoliberal: análisis crítico del discurso de la misión, visión y metas ${ }^{i}$
}

The discursive orientation of the Colombian public university in the context of neoliberal globalization: critical discourse analysis of the mission, vision and goals

Jorge E. Benavides B.

Departamento de Lingüística e Idiomas Universidad de Nariño (Colombia) 


\title{
Resumen
}

Este estudio analiza en forma crítica los documentos oficiales de 24 universidades públicas colombianas en su visión, misión y metas que integran buena parte de su razón de ser. Se utilizó un análisis crítico del discurso (ACD) teniendo en cuenta las categorías de función básicas de la Educación Superior. Se encontró que las universidades públicas han venido siendo consideradas a través de prácticas discursivas endógenas y exógenas más como organizaciones empresariales donde prima el concepto de la oferta de productos, bienes y servicios y donde la sociedad se considera el principal consumidor con necesidades latentes naturales y creadas. Se determinó la existencia de un proceso de recontextualización discursiva que representa a la universidad pública como una empresa orientada a la formación de mano de obra calificada para satisfacer las necesidades de la industria y del comercio en aras del crecimiento económico del país principalmente y a expensas de la formación de ciudadanos críticos.

Palabras clave: Educación Superior, misión, visión, metas, universidades públicas, Análisis Crítico del Discurso (ACD), recontextualización

\begin{abstract}
This study critically analyzes the official documents of 24 Colombian public universities in terms of the vision, mission and goals that make up a good part of their raison d'être. A critical discourse analysis (CDA) was undertaken considering basic Higher Education categories. It was specifically found that public universities have been considered through endogenous and exogenous discursive practices like organizational enterprises where the concept of the supply of products, goods and services is emphasized thereby considering the society as the main consumer with natural and created needs. The existence of a process of discursive recontextualization has been identified which makes the public university appear more like a business enterprise oriented to the formation of skilled labor to meet the needs of the industry, businesses and the economic growth of the country at the expense of the formation of critical citizens.
\end{abstract}

Keywords: Higher Education, mision, vision, goals, public universities, Critical Discourse Analysis (CDA), recontextualization 


\section{Introducción}

El contexto de tipo político, económico y tecnológico durante las últimas tres décadas ha hecho que la educación en general y la Educación Superior en particular se haya visto sujeta a presiones por el cambio de diferente índole y desde diferentes ámbitos y fenómenos contextuales. Uno de estos es el de la globalización que para Boltanski y Chiapello (2002) surgió como fenómeno discursivo acelerado particularmente por la caída del Muro de Berlín en 1989 y la consecuente desintegración de la Unión Soviética cuando se rompe el balance de la hegemonía política mundial. Otro fenómeno se da por el veloz desarrollo de las telecomunicaciones, la informática, telemática en gran parte del mundo que han catalizado la sociedad de la información y la sociedad en red (Castells, 1998). Un tercer fenómeno contextual implica a las políticas económicas neoliberales que surgieron como respuesta al modelo fordista, al del estado del bienestar y al keynesianismo, con lo cual se desestimó lo social (Sádaba Rodríguez, 2002). Tanto la globalización, como el neoliberalismo y el desarrollo de las nuevas tecnologías de la información y la comunicación han concurrido como factores de cambio en casi todos los campos del quehacer humano. Consecuentemente, la teoría neoliberal se ha presentado como la versión más avanzada de la teoría capitalista en la que se relacionan estrechamente la globalización (globalización económica) con la inmediatez tecnológica y su innovación constante. Esta teoría había recomendado, entre otras cosas, la liberación de los mercados (libres y abiertos a las fuerzas de la oferta y la demanda), la reducción del control estatal y la privatización de entidades de servicios públicos como la salud, la educación y el transporte (Fox, 2004). Se ha dado así un giro histórico para que las grandes empresas transnacionales controlaran gran parte de la economía internacional, los medios tecnológicos de comunicación y el dominio político en la configuración de la historia reciente de la humanidad en la que se puede hablar tanto de un determinismo político-económico (Yang, 2003) como tecnológico (Castells, 1998) que ha afectado instituciones tan importantes como la educación.

El presente estudio analiza el discurso de misión, visión y metas de la universidad pública que representan discursivamente la razón de ser, los valores, principios, propósitos y acción de la misma. Estos están en relación directa con su identidad y credibilidad ante la sociedad o comunidad determinadas (Marginson, 2007). Por otra parte, estos hacen parte de los factores establecidos en los lineamientos para la Acreditación Institucional de las universidades colombianas previstos y exigidos por el gobierno para cuyos 
procesos se ha enfatizado "la coherencia entre sus propósitos declarados y las acciones tomadas para llevarlos a cabo" (Lineamientos para Acreditación Institucional, 2014 p. 6).

\section{Marco histórico y contextual}

El nuevo modo de producción de la información y del conocimiento que Poster (1990) denomina 'modo de información' análogo históricamente al modo de producción industrial nació de la capacidad de procesamiento simbólico y ha significado nuevas formas de saber, transformando los modos de control y eventualmente de poder. La sociedad de la información y del conocimiento que surge con el concepto de la economía del conocimiento apoyada tecnológicamente en el contexto de la globalización se ha estado convirtiendo en la razón hegemónica del momento planteándose la paradoja de la sociedad en red en el sentido de que esta desconecta implacablemente a personas, instituciones y sociedades que no se ajusten a lo que la razón hegemónica del mercado considera como base del desarrollo (2000, Chomsky y Dieterich, 2002, Martín-Barbero, 2000). Además, el proceso continuo de prácticas discursivas de tipo colonial en la época moderna produce las perturbaciones del sistema sobre la sociedad en forma de 'colonización del mundo de la vida' donde prevalecen los imperativos del mercado, la economía y el capital que se ejercen priorizando el lenguaje técnico comercial y empresarial de la globalización a expensas de la función comunicativa (Habermas y Blazek, 1987; Habermas, 1992).

El surgimiento de la universidad global y de la universidad virtual ha sido la preocupación de los últimos años en la historia reciente del cambio educativo dadas las características de mega universidades que han adquirido recientemente algunas instituciones con alto poder tecno-económico, vinculadas discursivamente como grandes 'empresas educativas' y en asocio a corporaciones multinacionales. En este sentido, muchos analistas de la educación superior se han preguntado sobre el rediseño de las metas de la universidad en tanto esta se dispone a servir las necesidades de la economía (Scott, 2000). Además, se ha cuestionado el hecho de que la universidad sirviera principalmente metas e intereses ajenos a los que fundamentalmente habían sido diseñados como humanísticos, privilegiando en el primer caso el pensamiento enfocado a la eficiencia, el costo-beneficio de las inversiones y la estandarización, por encima de los de una institución libre, autónoma encargada 
especialmente de la formación del pensamiento crítico, autoreflexivo y a la vez creativo (Wendt, 1994).

Estas transformaciones han podido orientar un nuevo paradigma de organización educativa a la manera de una empresa económica que produce y ofrece bienes y servicios. La cultura, es decir, el sistema de creencias y de códigos históricamente producidos en la sociedad se han visto afectados por el nuevo sistema económico neoliberal, especialmente desde finales del siglo pasado. Cambios que para Castells (1998) son más que evidentes con los avances tecnológicos donde se enfatizan sus cualidades interactivas de acceso fácil, rápido y al 'alcance de todos', especialmente cuando se está conectado a la red principio de la universidad virtual. Lo anterior indica que el cambio es inicialmente de significados, por consiguiente, discursivo y eventualmente un fenómeno intertextual (Grant, 2005).

\section{Marco referencial}

\section{Creencias, valores y razón de ser}

Las instituciones en general como un conjunto de personas que buscan un fin y tienen unas funciones específicas trabajan en equipo como miembros de una misma organización. Todo el esfuerzo y el funcionamiento de sus integrantes están orientados a conseguir el propósito y razón de ser de la institución en lo que generalmente se denomina discursivamente la misión, la visión y las metas institucionales que tendrían que compartirse también desde el ámbito de cada integrante. En el nivel educativo estas responden a las cuestiones de la identidad, de las creencias, de los valores, y de las acciones o procedimientos.

Adicionalmente, estos elementos suelen verbalizarse explícitamente a nivel institucional con el fin de identificar y comunicar los valores de la institución a través de cada uno de sus actores dentro de la sociedad a la que sirven. La razón de ser de una institución que involucra sus creencias, valores, actitudes y comportamientos constituye el eje central sobre el cual gira el accionar de sus diferentes miembros. Consecuentemente, lo que la institución hace (misión), lo que desea ser (visión) y lo que desea alcanzar (metas) se traducen explícitamente en declaraciones textuales que constituyen la guía de navegación de la misma tanto para sus miembros como para la sociedad. 


\section{Misión}

Según lo anterior, la misión de una institución, concepto originalmente traído desde el ámbito empresarial (Drucker, 1974), es una declaración de propósito de la institución desde donde se pueden inferir sus valores, principios y creencias acerca del tipo de educación que contempla. Una apropiada declaración de la misión de la institución que represente la realidad de su existencia es un primer signo del éxito y del compromiso de sus actores.

En el nivel estructural, generalmente la extensión textual de la misión (como también de la visión) es usualmente de una sola declaración corta que condensa lo anteriormente mencionado. Sin embargo, no existe una regla específica al respecto no obstante tener la referencia de otras lenguas como el inglés donde se aconseja no más de 25 palabras (Brown, 1984) y que para el español podría estar en un rango de entre las 25 y 40 palabras y que usualmente se integran en una sola oración (Santana-Seda, 1975).

\section{Visión}

Esta se considera como la proyección de la institución o el deber ser de la misma. A diferencia de la misión que destaca principalmente lo que la institució es y lo que hace, la visión expresa lo que la institución será en su direccionamiento futuro, lo que quiere llegar a ser. Una visión del futuro de la institución es esencial en hacer saber a la sociedad lo que se espera de ella y el compromiso que de ello se deriva. Su extensión textual también se asimila a la de la misión.

\section{Metas}

Las metas son en sí los objetivos generales o fines propuestos por la institución. Para que éstas sean un empeño compartido es necesario que se desprendan coherentemente de la misión y la visión. A diferencia de las dos anteriores que son declaraciones cortas y sucintas las metas pueden desplegarse en mayor extensión. Sin embargo, al ser generales no podrían abundar en detalles específicos. En otras palabras, las metas son los mecanismos teóricos dispuestos como prácticas discursivas a través de los cuales se realizan tanto la misión como la visión y que dibujan en el mapa de orientación el curso de la institución. 


\section{Acreditación de programas y universidades en Colombia}

Durante la última década del siglo XX se llevó a cabo en Colombia el proceso de Acreditación de programas e instituciones de educación superior. La nueva relgamentación promovida por el Ministerio de Educación Nacional a través del CNA (Consejo Nacional de Acreditación) cobijó a las universidades en el contexto de la Ley 030 de 1992. Por consiguiente todos los programas de educación superior debían acreditarse previamente para mantener su registro calificado y poder funcionar. El decreto 272 de 1998 del Ministerio de Educación Nacional y los lineamientos para la acreditación previa de los programas de educación se empezaron a trabajar e implementar paralelamente con los planes de desarrollo institucionales.

Ante la sospechosa calidad de los programas de pregrado ofrecidos, el incremento de la demanda y la ineficaz oferta de programas actualizados y adecuados a los requerimientos sociales, según el Ministerio de Educación Nacional, el Consejo Nacional de Acreditación había definido los siguientes propósitos, entre otros:

- mejor cualificación de la educación superior

- autoevaluación permanente

- certificación de la calidad ante la sociedad

- solidez administrativa y académica de la educación superior

En este contexto, las universidades se dieron a la tarea de revisar y actualizar las políticas orientadoras de su acción educativa y administrativa. Esto se plasmó en documentos oficiales orientadores principalemente de la actividad académica, investigativa y de proyección social como los proyectos educativos institucionales, los planes de desarrollo institucionales a nivel general y los proyectos educativos de los programas a nivel particular.

\section{Antecedentes históricos de la misión y visión}

En el contexto mundial la exigencia de la misión fue un producto de las reformas educativas en educación superior en el Reino Unido y seguidamente en Los Estados Unidos. Esta reforma de finales de la década de los ochenta trajo control estatal de las universidades y se orientó especialmente para fines de rendición de cuentas sobre la financiación y el presupuesto otorgados. Esto se ha considerado como un movimiento hacia la introducción de una visión de 
manejo empresarial y mercantil en la universidad empezándose a exigir productos verificables que justificarían un determinado tipo de financiación estatal. Las declaraciones de misión que incluían la razón de ser y los propósitos de las universidades se tendrían como referencia para determinar el cumplimiento de las metas $\mathrm{u}$ objetivos en tanto indicadores de logro y eficiencia con los fondos públicos para la docencia y la investigación (Connell \& Galasinski, 1998).

Según Peeke (1994) entre las exigencias a las universidades por parecerse administrativamente más a empresas de negocios o proveedores de bienes y servicios estaban las de eliminar fondos para el sostenimiento al incremento del número de estudiantes; cambiar el modelo de la institución como proveedora de educación a ser la institución centrada en atraer mediante la oferta a los consumidores de sus productos educativos. En este sentido la universidad estaría más orientada a las reglas del mercado y sujeta a las leyes de la oferta y la demanda. La presión de la industria por tener mano de obra mejor calificada también hizo que se incrementara el énfasis en el desarrollo de competencias para un tipo de trabajo flexible y adecuarse a los requerimientos de una gran variedad de empleadores que requerían especialmente empleados eficientes y competitivos.

Lo anterior tuvo un impacto directo en el rediseño de los currículos ya que estos tenían que estar de acuerdo con las necesidades de la economía y relacionarse con la futura fuerza laboral, es decir, las necesidades a suplir fueron en realidad las de la industria y los negocios con una urgente necesidad de mano de obra calificada. Las necesidades por satisfacer fueron en su gran mayoría aquellas derivadas de la educación de tipo profesionalizante que enfatizaban los gobiernos de los países desarrollados (Reino Unido y Estados Unidos) y que organizaciones como la OCDE y el Banco Mundial exigían a las universidades para mejorar la productividad económica como indicador de desarrollo y de progreso nacional. Lo que importaba no era lo que necesitaban y deseaban los estudiantes como una posibilidad de formación sino lo que ofrecían los currículos promulgados por las diferentes agendas políticoeconómicas de turno (impulso de las políticas macroeconómicas de Margaret Thatcher y Ronald Reagan en la década de los ochenta). Estas fueron determinantes en la transformación de las universidades con el incentivo de la formación del capital humano y de la fuerza laboral necesarias para el desarrollo sostenible de la economía (Jenkins, 1995).

La transformación de las universidades en instituciones de educación superior como organizaciones de tipo mercantilista orientadas a la producción 
de bienes y servicios fue un requisito para justificar la financiación de las mismas a las que, a través de su nueva estructuración, se podría exigir resultados y productos visibles como una forma de justificar el uso eficiente de los recursos estatales. De la misma manera, la evaluación de las universidades como un proceso de rendición de cuentas era anteriormente engorroso y financieramente desgastante si no se cambiaba a un esquema de disciplina organizativa empresarial (Peeke, 1994). El efecto de estos cambios hizo que las universidades como instituciones educativas ya no obedecieran al concepto tradicional de la 'idea de la universidad' pregonado por Alexander von Humboldt, Henry Newman y Karl Jaspers, y más recientemente por Jurgen Habermas. Ahora se tendrían universidades orientadas al trabajo, al campo laboral profesionalizante administradas más como corporaciones con planes estratégicos.

\section{Discurso de la educación superior y género}

En la educación superior y en el contexto de la influencia de procesos históricos como el capitalismo, la globalización y las políticas neoliberales en la sociedad se evidencia un aumento apreciable de un individualismo que fractura ideológicamente la solidaridad que se requiere para un tipo de lucha y compromiso en el mundo real. Desde esta perspectiva se establece la presencia de "poderosas transformaciones ideológicas que ocurren en nuestras sociedades [...] que son en realidad parte de un 'proyecto educacional' a gran escala" (Apple, 1999, p. 4). Según Apple, además de estos cambios, y alrededor de la posición política, se promueve un proceso de modernización como movimiento neoconservador donde se incluyen, neoliberales, neoconservadores, populistas autoritarios, y la nueva clase media móvil. Esta visión igualmente considera a los estudiantes como 'capital' en un mundo de una competitividad económica intensa en la que tienen que proveerse de habilidades y competencias técnicas para funcionar eficientemente. A esto se añade la crítica de Paulo Freire de la clase dominante que “... sorda a las necesidades de una lectura crítica del mundo, insiste en la capacitación puramente técnica de la clase trabajadora, con la que esta se reproduce como tal...” (Freire, 1996, p 97). La posición normal, o de sentido común es que la educación sea más relevante con la tecnología, la producción de bienes y servicios y el desarrollo económico. Esto ha servido como clara justificación para que las instituciones educativas (especialmente las privadas) exhiban tecnologías de punta y con alta inversión en ciencia y tecnología que ha marcado la ruta a seguir de las instituciones públicas. 
La propaganda ideológica, que para educadores como Paulo Freire, viene utilizando la clase dominante es precisamente e irónicamente la 'desaparición de las ideologías' y una nueva historia sin clases sociales y sin luchas dentro del contexto de la postmodernidad. Este es el éxito del 'sentido común' dentro de las democracias capitalistas actuales que 'naturalizan' las prácticas de un discurso pragmático que promueve la desaparición de antagonismos como lo es en una democracia 'saludablemente capitalista'. Sin embargo, es desde la perspectiva del educador progresista que Freire ve la educación; no desde de la concepción mecanicista de la historia sino desde la construcción de la historia por los hombres y mujeres; no como piensan construirlo las fuerzas neoliberales a través de cambios puramente ideológicos. Por consiguiente, la lectura del mundo debe ser una lectura crítica (Freire, 1996, p. 100).

El papel del discurso en la sociedad de finales del siglo XX ha tomado una importancia central en cuanto al cambio sociocultural, transformación que en el campo educativo se investiga en términos de prácticas discursivas, su relación con las prácticas sociales. Fairclough, (1993) al analizar el discurso de la universidad en Europa y como caso particular del Reino Unido encontró procesos de 'mercantilización' de la universidad en sus prácticas discursivas que comprende nuevas combinaciones de géneros, discursos y textos tanto en su forma como en su función o significado. La tecnologización del discurso es el foco de atención que integra el movimiento de prácticas como parte del cambio social y que constituye un indicador sensible de las tensiones sociales en su proceso de evolución histórica.

Más recientemente y el contexto de la Unión Europea también analizando los discursos de políticas de educación Wodak y Fairclough (2010) encontraron patrones que sugieren una marcada influencia de sectores externos a la educación con una indudable influencia interdiscursiva, es decir, el discurso de la educación afectado y matizado por discursos de la economía de mercado, de la globalización y de políticas neoliberales orientadas a la retórica de la competitividad cada vez más frecuentes:

Cuando se analizan los más recientes documentos, es obvio que después de haber transformado las estructuras de la universidad, la implementación del proceso de Boloña sólo empezó alrededor del 2005, acompañado del discurso de la competitividad, la retórica de la globalización, la economización y la estandarización requerida (p. 31).

Se entiende desde esta perspectiva el género de los discursos oficiales sobre política universitaria con una serie de prácticas discursivas exhortativas y 
factuales típicas de un nivel institucional, responsable de políticas tendientes a promover el cambio, la necesidad, la deseabilidad y valor de la acción en sus declaraciones. Estas en la actualidad se han acordado en forma endógena en lo particular (educación) y exógena en cuanto a las políticas de apertura económica e internacionalización como medio para prepararse a las exigencias del contexto de lo global.

También incluye políticas institucionales de regulación, de legitimación y control de nivel global que giran en torno a conceptos extendidos de políticas económicas neoliberales a través de proyectos políticos mundiales como el de la globalización. Esta tendencia institucional que se ha hecho más evidente a partir de la última década del siglo $\mathrm{XX}$ en lo que se conoce como 'el nuevo espíritu del capitalismo' (Boltanski y Chiapello, 2002), y las consecuencias o afectaciones de la globalización (Giddens, 2000) ha sido especialmente influenciado por aspectos cambiantes de la economía de mercado, conceptos de reingeniería y gestión empresarial para la evolución económica exitosa y próspera de los negocios (Kanter, 2002), aspectos de influencia en los campos más tradicionales como el de las políticas institucionale en educación.

\section{Marco teórico-metodológico: Análisis Crítico del Discurso}

El ACD como enfoque teórico y metodológico tiene como uno de sus orígenes el trabajo realizado por la linguiística crítica (cfr. Fowler et al, 1979) que pretende mediante el análisis lingüístico estudiar las relaciones entre los signos, los significados y las condiciones históricas que dominan como fenómeno discursivo en un determinado contexto social. Más tarde, la linguiística crítica se integra al análisis del discurso que considera el lenguaje como un fenómeno socio-semiótico (cfr. Halliday, 1982 [1978]). Sin embargo, el ACD surge como campo de investigación desde los esfuerzos interdisciplinarios de Ruth Wodak, Norman Fairclough y Teun van Dijk, entre otros, en los inicios de la década de los noventa como un producto de la teoría socio-crítica, de la linguística crítica y el análisis del discurso.

En el ACD el lenguaje es el sistema por medio del cual se establecen relaciones de tipo social y es en el uso de la capacidad de expresión y acción donde se lo considera como una forma de práctica social (Fairclough, 1989, 1992, 1995). Este mismo uso es también un ‘modo de acción' situado histórica y socialmente, es decir, que está configurado socialmente y que a la vez también configura el aspecto social en una relación dialéctica. En este sentido y dada la naturaleza social del lenguaje se ha desarrollado una teoría social del 
discurso no únicamente centrada en su funcionamiento lingüístico sino en la relación de las prácticas discursivas con lo social. La siguiente figura muestra esta relación.

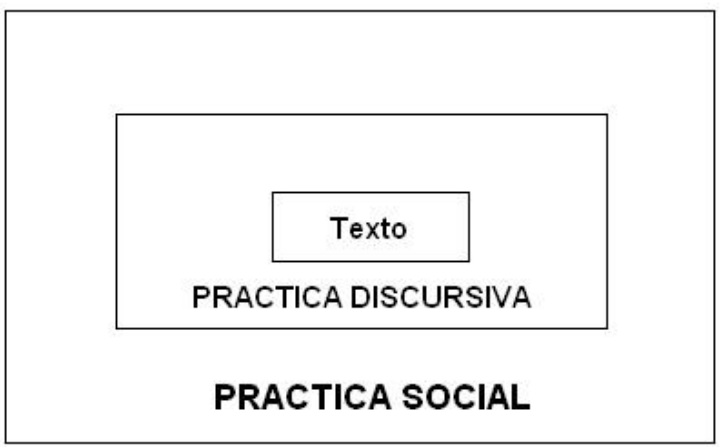

El lenguaje como práctica social (Tomado de Fairclough, 1992 p. 73)

El lenguaje en su uso (o discurso) se plantea como una forma de práctica social a través de las prácticas discursivas en las que se materializa como texto, lo cual implica, en primer lugar, que el lenguaje es parte de lo social y no algo externo a él, y que cuando se usa el lenguaje se lo hace mediante formas que están determinadas socialmente y que a la vez tienen efectos sociales. Además, el lenguaje es parte de la sociedad donde las discusiones y debates (orales y escritos) en política, por ejemplo, son el centro de una buena parte de la actividad social.

El 'modo de acción' que constituye el uso de la lengua implica un acontecer dentro de lo social; esto es, el contexto en el cual se sitúa un evento discursivo lo hace también históricamente situado. De esta manera, filósofos y lingüistas como Michel Foucault y Norman Fairclough han buscado explorar la función del lenguaje y la tensión creada entre lo que es socialmente configurado y lo que se configura en el lenguaje a diferencia de lo formalmente descriptivo como en el caso de la tradición estructuralista de la lengua. Así, el lenguaje es el vínculo semiótico que permite producir y renovar los significados culturales, los mensajes complejos de un contexto histórico en el cual se desarrollan la sociedad y sus prácticas. De esto se desprende que la historia de la humanidad es, en alto grado, la historia de la actividad semiótica como lo es de la actividad socioeconómica (Halliday, 1982). 


\section{Discurso e ideología}

En canto a la dimensión ideológica en los discursos, en la tradición del ACD se rechaza el criterio mediante el cual el lenguaje es una representación directa de la realidad social, libre de todo valor ideológico. El hecho de que toda representación está mediada o modelada por los sistemas de valor que se incrustan en el medio lingüístico cobra más preponderancia cuando necesariamente este último se realiza en un contexto históricamente determinado (social, político, económico) donde generalmente se asientan ideologías que funcionan dentro de los sistemas sociales y que se difunden, cambian o perpetúan a través de sus instituciones (Kress y Hodge, 1979; van Dijk, 1998; Wodak, 2003).

Siguiendo a Reboul, (1986) entre más implícita o disimulada sea la función real de las formas del texto mayor el efecto ideológico del discurso. La forma más efectiva de ideología es la que se encuentra en el trasfondo (implícita e inconsciente) y pasa desapercibida como parte de lo que se asume como de 'sentido común' en tanto no se la cuestiona y se da por supuesta (Fairclough,1989). De la misma manera, el poder se ejerce desde la ideología en el discurso en la forma como se presentan los contenidos, las relaciones que se establecen entre el autor (o productor) del texto y el intérprete y las identidades que se constituyen. Estas dimensiones de significado en el discurso están relacionadas con los valores formales del texto en cuanto a la experiencia del mundo, a las relaciones entre el autor y el intérprete y la expresión dentro del texto que caracteriza la dimensión descriptiva del mismo. En otras palabras, el objeto de la crítica de la ideología en el enfoque social del ACD es el de internarse reflexivamente en la textura del documento y en su discurso para detectar esas relaciones de poder e ideología inconfesas. Por otro lado, el concepto de ideología de tipo socio-cognitivo, radica en la forma cómo esta se expresa, cómo cambia y se reproduce a nivel institucional convirtiéndose en la base de las representaciones sociales compartidas por quienes componen un grupo. Esto significaría que, “... las ideologías permiten a la gente, como miembros de un grupo, organizar la multitud de creencias sociales acerca del caso, bueno o malo, correcto o incorrecto, según ellos, y actuar en consecuencia." (Van Dijk, 1998, p. 21).

Finalmente, el ACD intentaría determinar cómo las ideologías dominantes aparecen en diferentes formas en el discurso oficial de las universidades y mediante qué estrategias discursivas se integran al registro de los documentos que los configura como parte de un sistema de valores que se 
asumen desde el contexto de la globalización y las políticas económicas neoliberales del momento.

\section{Recontextualización discursiva}

El contexto en el que los textos (documentos) cobran vida y valor, se comprende principalmente en la forma histórica en la que intervienen y en sus relaciones con otros textos (intertextualidad) y otros discursos (interdiscursvidad) -que a menudo provienen de otros contextos- para producir el "enfoque histórico del discurso deudor de la teoría crítica, [que]...encuentra su punto focal en el campo de la política, ámbito en el que intenta desarrollar marcos conceptuales para el discurso político” (Meyer, 2003, p. 46). Además el concepto de intertextualidad es decisivo en el ACD puesto que es necesario el análisis de las relaciones entre los textos, sus contextos además de las características lingüísticas de su análisis. La característica del discurso se fundamenta en el hecho de que "Los discursos tienen raíces históricas y están entretejidos" (Ruth Wodak, 2003, p. 28). La red discursiva en la que se producen los textos y discursos es de innegable importancia en la consideración de la intertextualidad puesto que esta última se constituye en el punto de partida para el análisis de carácter histórico de los discursos en un espacio y tiempo definidos.

En otras palabras, La recontextualización discursiva determina cómo las prácticas discursivas y representaciones sociales utilizadas en un dominio y contexto se encuentran difusamente presentes en otros tipos de discursos: económico, político, tecnológico, educativo, etc. También es importante en cuanto a su determinación ideológica puesto que son las formas y sus significados los que se utilizan, provenientes de usos y contextos previos con representaciones modificadas en los nuevos. En otras palabras, este proceso ocurre generalmente dentro de lo que se denomina la 'colonización del discurso' donde las practicas discursivas de un contexto o dominio se utilizan consuetudinariamente en otro, modificándolo y eventualmente impregnándolo ideológicamente.

\section{El corpus, categorías, unidad de análisis y características textuales}

Para efectos de este análisis de utilizaron como fuentes los textos de la misión, la visión y metas de las 24 universidades públicas seleccionadas en 
representación de la mayor parte de regiones del país (Ver Tabal 1, abajo). Los documentos se los obtuvo principalmente de los documentos del Proyecto Educativo Institucional y del Estatuto General en formato PDF disponibles en las páginas web de estas instituciones. En cinco casos se visitaron las universidades para la solicitud directa de la información oficial (Universidad Nacional, Universidad Pedagógica Nacional, Universidad de Cartagena, Universidad Pedagógica y Tecnológica de Colombia, y Universidad del Valle).

Tabla 1: Universidades participantes

\begin{aligned} \hline & UNIVERSIDADES PÚBLICAS \\ \hline $\mathbf{1} &$ Universidad Nacional de Colombia \\ $\mathbf{3} &$ Universidad de Antioquia \\ $\mathbf{4} &$ Universidad del Valle \\ $\mathbf{5} &$ Universidad Industrial de Santander \\ $\mathbf{6} &$ Universidad de Córdoba \\ $\mathbf{7} &$ Universidad del Cauca \\ $\mathbf{8} &$ Universidad de Caldas \\ $\mathbf{9} &$ Universidad del Tolima \\ $\mathbf{1 0} &$ Universidad Tecnológica de Pereira \\ $\mathbf{1 1} &$ Universidad del Atlántico \\ $\mathbf{1 2} &$ Universidad del Quindío \\ $\mathbf{1 3} &$ Universidad de los Llanos \\ $\mathbf{1 4} &$ Universidad de Nariño \\ $\mathbf{1 5} &$ Universidad del Magdalena \\ $\mathbf{1 6} &$ Universidad Pedagógica Nacional \\ $\mathbf{1 7} &$ Universidad Pedagógica y Tecnológica de Colombia \\ \hline $\mathbf{1 8} &$ Universidad Surcolombiana \\ $\mathbf{1 9} &$ Universidad Popular del César \\ \hline $\mathbf{2 0} &$ Universidad de Cundinamarca \\ $\mathbf{2 1} &$ Universidad Francisco de Paula Santander \\ $\mathbf{2 2} &$ Universidad de Sucre \\ $\mathbf{2 3} &$ Universidad de la Guajira \\ $\mathbf{2 4} &$ Universidad Tecnológica del Chocó \\ \hline & \end{aligned}

Se hizo énfasis en la vigencia de estos tres tipos de discurso en cada documento y se utilizó la versión más reciente disponible. Se consideró que debería haber como base un acto administrativo (acuerdo) por las instancias respectivas, ya sea de la Oficina de Secretaría General o del Consejo Académico que respaldaran el documento.

El corpus estuvo compuesto de 24 declaraciones de misión, 24 declaraciones de visión, y muchas más declaraciones de metas de cada una de las universidades participantes como tipo de oraciones más cortas. La gran 
mayoría de declaraciones de misión y visión estaban dispuestas como oraciones extensas sin mayor estructuración de párrafo como en el caso de un ensayo. La mayoría consistió en declaraciones de una o hasta dos oraciones extensas.

Las categoría principales del análisis es el discurso de la misión, de la visión y de las metas a nivel general, sin embargo, el análisis se centró particularmente en las tres funciones sustantivas de la universidad como categorías particulares de análisis: la docencia, la investigación y la extensión (o interacción) social, la calidad de la educación, el lugar de las Ciencias Sociales, las Humanidades y las Artes y las relaciones de necesidad, de oferta y de demanda, entre otras. No todas las universidades tenían las declaraciones de la misión, la visión y las metas en un lugar destacado y fácil de identificar en sus documentos. En muchos casos estaban haciendo parte de los informes de autoevaluación de los factores de acreditación institucional o de los programas o conformando otras categorías. Algunas de las metas estaban incrustadas como propósitos o como objetivos y fines y enmarcados en las funciones de la universidad como la teleología institucional. La fecha de publicación de dieciséis de los textos databa de la primera década del siglo XXI (2000-2008) con siete casos de la segunda década (2008-2016). Solo la Universidad Nacional contaba con una misión y visión que databan de postrimerías del siglo XX. Esto manifiesta la muy reciente construcción o actualización de los textos debido especialmente a los procesos de acreditación de los programas y a la acreditación institucional.

La unidad de análisis fue la oración como unidad mínima de sentido y como parte integral del discurso desde los documentos oficiales como género de políticas institucionales en educación en el nivel general y en tanto formas de acción e interacción en el acontecer de las prácticas de la universidad en particular. Sin embargo, dentro de esta unidad de análisis se recurrió a la identificación de términos y conceptos determinados por frases y palabras para destacar la importancia de un determinado significado.

La longitud en términos de número de oraciones y de palabras por oración fue muy diversa y contrasta con la moderada regla de entre 25 y 40 palabras por oración. Tomando como base una sola oración como declaración para la misión, la mínima fue de 36 para la Universidad del Cesar seguida de la Universidad de Nariño con 49 y las máximas fueron de 120 y 110 para la Universidad del Quindío y de la Guajira, respectivamente. Para la visión, teniendo el mismo criterio, las mínimas fueron para la Universidad del Atlántico con 16 seguida de la Universidad del Cauca con 39. Las mayores 
fueron para las Universidades Surcolombiana y del Quindío con 80 y 64, respectivamente.

\section{Analisis critico del discurso de la mision, vision y metas}

A continuación se describe y ejemplifica para efectos del presente análisis inicialmente una característica importante de práctica discursiva como la identidad institucional. Existen tres clases de declaraciones distinguibles de misión, donde se aprecia el foco o énfasis semántico: la institución, el contexto y la misión como las partes que elaboran la identidad. En la primera clase el foco es la institución y por consiguiente esta se constituye en el agente asumiéndose una mayor carga de significación en la identidad institucional.

\section{La Universidad $\mathbf{X}$ [7 ocurrencias]/forma/propicia/asume/educa/orienta/}

La segunda clase describe a la universidad desde su contexto de situación e identidad seguida de su actividad.

\section{La Universidad $\mathbf{X}$ es una institución $\mathbf{Y}$ [10 ocurrencias] que desarrolla/que forma.}

La tercera clase está centrada en la misión, aunque en forma indirecta con una menor carga de identidad.

La Universidad $\mathbf{X}$ tiene como misión/propósito/fin [7 ocurrencias] educar/formar/la formación/la transformación.

$\mathbf{X}$ sirve denomina la instutción, sujeto o agente de la acción o proceso, $\mathbf{Y}$ para los complementos u objetos de la acción. Entre corchetes rectangulares las ocurrencias de la estructura anterior y en cursvia las diferentes formas de ocurrencia.

\section{Docencia, investigación y extensión}

Para la misión, es la institución la que actúa como agente en procesos como cumplir, influir, generar, difundir, orientar el 'conocimiento' como complemento y especialmente para (el proceso más común) formar estudiantes, egresados y particularmente profesionales altamente cualificados.

El clásico enunciado declarativo de la misión donde coocurren estas tres funciones es generalmente del siguiente tipo, 
La Universidad $\mathbf{X}$, como institución pública, mediante el cumplimiento de sus funciones sustantivas de docencia, investigación y extensión, y de su proceso de internacionalización, forma profesionales competentes en distintas áreas del conocimiento...

Menos frecuente (tres casos), pero más inclusiva y personalizada es la siguiente,

Somos una Universidad pública con talento humano cualificado y reconocimiento social, que mediante actividades de docencia, investigación y extensión, forma profesionales idóneos, críticos e íntegros, capaces de articular el conocimiento científico, tecnológico y cultural con el desarrollo socioeconómico sostenible...

Es clara la representación de la universidad como agente que 'forma profesionales' en una relación proceso-producto en el contexto de las políticas de globalización internacional de la educación en el primer caso y para desarrollo socioeconómico sostenible en el segundo. Se destaca el uso de competentes para los profesionales como parte del desarrollo de habilidades o competencias prácticas e instrumentales en la diversidad de áreas de conocimiento y en el segundo caso se mitiga el componente profesionalizante con el uso de críticos pero que contrariamente relaciona el conocimiento con el desarrollo socioeconómico que se califica como sostenible.

En la visión, la investigación adquiere una posición más central y aparece como el deseo, el querer y el deber ser de la universidad pública colombiana para el futuro donde todos anhelan sobresalir a pesar del acervo y centralidad de la docencia en la mayoría de las universidades públicas. Así lo manifiestan las 29 ocurrencias del término y en casi la totalidad de los casos en forma independiente.

La mayoría de las declaraciones de visión representan a la universidad como la institución que busca el reconocimiento social sustentado en la investigación como el eje del deber ser. Se utilizan calificativos que le otorgan valor explícito como, alta calidad, de la más alta calidad, central, exitosamente, y con recurso a nombres o sustantivos como; alto prestigio, ser líder, referente obligado, excelencia investigativa, con pertinencia y con reconocimiento. Consecuentemente, el deber ser de la universidad se destaca en la investigación como práctica discursiva que agrega valor explícito en los niveles institucional, nacional, regional, internacional y también global como lo indican los siguientes casos. 
En el año 2016, seremos la principal universidad de investigación del país y una de las mejores de América Latina, con pregrados y posgrados de excelencia académica internacional...

La Universidad $\mathbf{X}$, como una de las más importantes instituciones públicas de educación superior en Colombia en cobertura, calidad y diversidad de sus servicios, aspira a consolidarse como una universidad de excelencia, pertinente, innovadora, eficiente, competitiva, con proyección internacional y de investigación científica.

La Universidad habrá consolidado una política de articulación global que le ha permitido incrementar de manera significativa los resultados de sus procesos misionales mediante la cooperación con instituciones educativas y de investigación de alto prestigio...

Universidad de alta calidad, líder al 2019 en la región y en el país, por su competitividad integral en la docencia, investigación, innovación, extensión y gestión para el desarrollo humano con responsabilidad e impacto social, inmersa en la comunidad internacional.

La Universidad $\mathbf{X}$ en el año 2015 será reconocida internacionalmente como una institución de alta calidad académica y excelencia en sus proyectos educativos, de investigación y proyección social,

En el año 2022 seremos una institución modelo de desarrollo académico transdisciplinar, articulada a la realidad regional, nacional y global, líder en la formación, la investigación y la proyección social para la producción de conocimiento, dirigida a la excelencia.

La Universidad $\mathbf{X}$ estará reconocida institucionalmente en el ámbito nacional e internacional, por sus actividades de docencia, investigación y de extensión de alta calidad, excelencia académica y pertinencia social.

Al 2020 la Universidad $\mathbf{X}$ será reconocida nacional e internacionalmente como miembro de la sociedad del conocimiento, por la calidad de sus procesos académicos de docencia, investigación y extensión, y, los articulará con el desarrollo socioeconómico de Colombia.

Como se puede apreciar existe una intensificación explicita de la calidad y valor de la investigación como estrategia para buscar el reconocimiento de la institución. El valor explícito e implícito se manifiesta en el uso de lo internacional, lo mundial y lo global que aparecen como aspectos muy deseables a lograr. El valor deóntico que imprime el uso del futuro en las declaraciones también se destaca como forma discursiva de afirmación o como 
logro futuro de la institución. Discursivamente las valoraciones explícitas y asumidas que despliega la universidad en cuanto a la función misional de la investigación juntamente con la docencia y la extensión la representan en la práctica discursiva como un hecho posible o incluso real.

Lo anterior no es muy diferente al tipo de discurso de las organizaciones empresariales que en su misión, visión y metas intentan vender su imagen para un determinado sector del mercado ofreciendo su imagen corporativa y sus productos, bienes y servicios que desea se adecúen a las necesidades de los usuarios, clientes o consumidores. Por otro lado, y como lo analiza Fairclough (1993) y Wodak y Fairclough (2010) en tanto 'mercantilización' de la universidad la referencia a términos como servicios, la calidad, la competitividad, la eficiencia, y lo global, insinúan una recontextualización del discurso de la universidad desde sus documentos orientadores.

En cuanto a las metas de la universidad, la investigación aparece principalmente como algo que se intenta promover y fortalecer intuyendo que esto necesita orientación, capacitación y consolidación. Sin embargo, también se presentan metas más ambiciosas que no necesariamente encajan con la realidad del enfoque docente de pregrado de la mayoría de las universidades públicas a excepción de unas pocas que se consideran dentro del enfoque de la investigación (maestrías y doctorados).

Los siguientes casos podrían presentar algo en común con lo expuesto de la valoración que se da a la investigación precisamente con el supuesto que todavía no se tiene lo que se quiere alcanzar, y que posicionaría altamente la imagen de la universidad pero que desde ya se lo valora explícitamente en alto grado.

Hacer de la investigación un eje central de la actividad universitaria y propiciar su integración con los programas de formación.

Realizar Investigación de Alta Calidad Orientada al Desarrollo Científico y Conducente a Innovaciones Tecnológicas, $\underline{\text { Sociales, Económicas, Culturales y Políticas }}$ Pertinentes con el Desarrollo del País.

El uso del artículo indefinido un en el primer caso mitiga le centralidad o énfasis dado a la investigación, a expensas de la docencia y la extensión, por ejemplo. En el segundo se da valor explícito a la investigación de alta calidad, pero aparece como excluyente al estar orientada al desarrollo científico. Valdría la pena preguntarse si este tipo de investigación es la que conduce a la lista del tipo de innovaciones que se desean lograr. Implícitamente con el listado de 
innovaciones se da valor a toda esa gama de posibilidades creando el efecto discursivo de completitud de las metas institucionales. Sin embargo, tanto la investigación en educación como formación de educadores brilla por su ausencia con respecto a esta función en las declaraciones de las 24 universidades. Esto evidencia la orientación profesionalizante de la universidad con el desarrollo material según las observaciones de Aronowitz, (2001) y Giroux, (2001).

\section{Conocimiento}

Generalmente se asocia a la universidad como la principal entidad social poseedora, generadora y difusora del conocimiento en los diferentes campos, sin embargo, procesos verbales como difundir/producir cuya ocurrencia se esperaba no se utilizaron con conocimiento como complemento directo a excepción de un caso. En varias instancias se encuentra en forma más personal,

La Universidad $\mathbf{X}$ genera/transforma/transfiere/contextualiza/aplica/gestiona/innova e intercambia el conocimiento en ciencia, tecnología, arte y cultura.

Pero también ocurre el recurso a la nominalización como complemento del verbo:

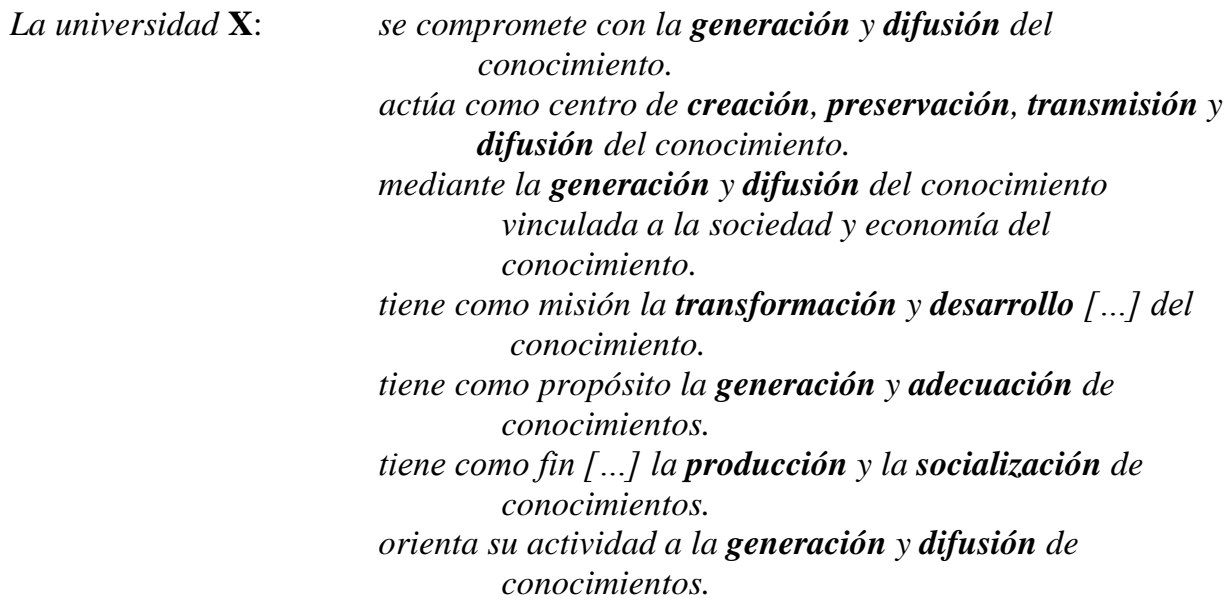

El efecto principal de las anteriores expresiones nominalizadas es la omisión del sujeto, es decir, no se sabe quién realiza la acción y consecuentemente al actor principal se lo minimiza ya sea por razones ideológicas o se omite para efectos de asociarlos a la institución como tal a expensas de los actores 
principales, los estudiantes, los docentes, los investigadores, los docentesinvestigadores y la comunidad educativa en general. En las declaraciones de visión se evidencia el mismo proceso en tanto la universidad se asocia a la generación/adecuación/globalización/producción/gestión/aplicación del conocimiento.

Como se manifestó anteriormente, el efecto principal de la nominalización es la minimización del sujeto y por ende del agente de la acción y la acción misma como proceso. Se expresa solo el objeto o complemento sobre el cual recae la acción, sin embargo, tanto el sujeto agente como la acción han sido obscurecidos.

Con las metas ocurre lo contrario ya que el uso del proceso o acción verbal es el principal aspecto de lo que se realiza. En posición tópica ocurren, por ejemplo, fortalecer[30], promover[27], contribuir[20], consolidad[12], desarrollar[15], formar[15].

Estos procesos, aunque en forma impersonal son los que la Universidad /busca, intenta/ realizar como metas a largo plazo. Estas acciones provienen de la Universidad mayormente como sujeto y agente hacia o sobre los complementos o beneficiarios como son los estudiantes y los docentes. Estas se declaran como nominalizaciones mayormente,

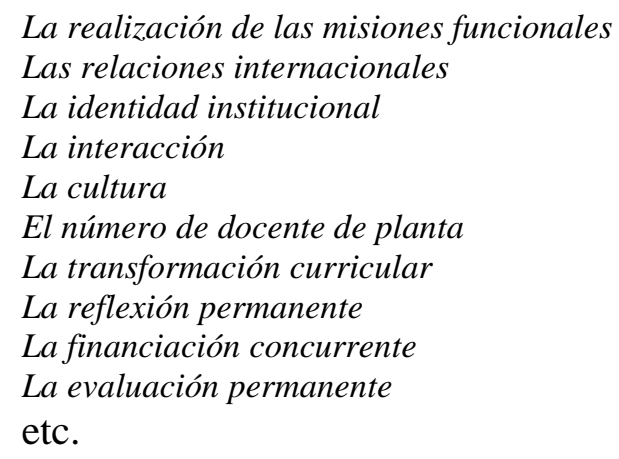

Sin embargo, son muy pocas las instancias de procesos en los que se infiere la participación y compromiso de otros actores como sujetos y agentes de la acción, a excepción de la parte administrativa. Ocurren procesos en forma de infinitivos (impersonales):Garantizar[1], ofrecer[2], mejorar[3], impulsar[4], ampliar[4]

Con complementos objeto como

El cumplimiento de su misión y visión.

Un servicio público de educación superior de acuerdo con los principios, derechos y deberes consagrados... 
Programas de educación ... para el trabajo y el desarrollo humano. La modernización pedagógica y curricular acorde con las demandas sociales. La cobertura mediante la oferta de nuevos programas académicos. Los aportes de la academia a la sociedad del conocimiento.

Estos son aspectos que obviamente implicarían un compromiso mayor para la universidad y por ende no sobresalen en términos de ocurrencias observables y más bien aparecen minimizados. Contrariamente a lo anterior, en uno de los procesos se utiliza como objeto directo a egresados: como complemento de propósito del proceso producir:

... para producir egresados que contribuyan con el desarrollo de innovaciones en la ciencia la tecnología y la cultura.

La anterior declaración enfatiza a egresados como objeto de producir considerando a las personas un producto de la institución dentro de un proceso de tipo material traído del campo de la industria y aplicado a los individuos y a la sociedad en general. Esto difiere del proceso formar que como misión tiene una determinada universidad para con los profesionales, ciudadanos, personas, hombres y mujeres, comunidades académicas, etc.

Con estas, o más opciones discursivas disponibles de representar los procesos (eventos) se origina la posibilidad de destacar o excluir determinados elementos de un evento discursivo, de imprimirles o quitarles valor según las formas de representación que se desplieguen en el texto. Este proceso de nominalización en general, según el $\mathrm{ACD}$ es muy frecuente y productivo en los discursos de políticas en educación (Fairclough, 2003). Por otro lado, como manifiestan Graham (2001) y Lemke (1995), la ocurrencia de la nominalización es una característica de los discursos de tipo político, científico y tecnológico donde existen altos niveles de abstracción y generalidad en los conceptos que se manejan. En el presente caso se ha encontrado que la nominalización es un recurso productivo, ya que permite generalizar y abstraer de eventos particulares series y conjuntos de eventos generales, como el caso del discurso técnico (Halliday \& Martin, 1993) como también del discurso de políticas en educación. 


\section{Calidad de la educación}

La calidad como valor dado a los procesos educativos está presente en forma directa tanto en la misión, como en la visión y las metas de manera creciente. Se intensifica con la calificación 'alta' tanto en nominalizaciones,

Formación de personas

Formación de profesionales

como en nombres compuestos o frases nominales,

Profesionales
Universidad
Institución
Extensión
Programas de pregrado y posgrado
Maestrías y doctorados
Cultura y gestión

Una intensificación de mayor grado se realiza en 'programas de la más alta calidad'. La excelencia académica[5] es otra de las adjetivaciones positivas en este tipo de discurso.

Nuevamente, como en los servicios y productos que ofrece la Universidad se agrega valor a los nombres para hacerlos deseables a quienes se ofrecen, los 'consumidores'. Esta práctica discursiva está muy relacionada con el discurso publicitario en el que se promocionan productos, bienes y servicios. Fairclough (1993) describía ya desde finales del siglo XX este proceso y lo relacionaba con el nuevo concepto de 'mercantilización' de la universidad en el Reino Unido, proceso discursivo que se ha extendido a nivel global en la educación superior.

Otro tipo de calificativos utilizados incluyen buenos, mejores y hasta excelente, alto(a), significativo, y flexible orientados a nombres como prácticas, organización, calidad, nivel, sentido, estilos, aprendizaje, etc.

Las universidades se distinguen ahora por estar representadas a través de prácticas discursivas como organizaciones que se destacan por la oferta de productos de alta calidad y de excelencia a la manera de empresas comerciales que ofrecen 'excelentes' productos y servicios a aquellos que los requieren o necesiten y puedan adquirirlos. Sin embargo, es necesario resaltar que solo dos universidades declaran en sus metas el propósito de lograr la acreditación de alta calidad para sus instituciones, lo cual relaciona implícitamente a dos 
agentes externos, El Ministerio de Educación Nacional y El Consejo Nacional de Acreditación a este proceso.

Mucha de la búsqueda de la alta calidad y la excelencia tanto en la misión como en la visión y las metas de las universidades está dada mayormente sin mencionar los beneficiarios como complemento de la acción, justificando la excelencia en si misma o buscándola como un fin institucional. Se podría asumir, al no estar estos explícitos que la excelencia se justifica para la sola institución en tanto agente administrativo. El siguiente es uno de los muchos casos de la misión donde solo se puede inferir los beneficiarios,

La universidad $\mathbf{X}$ fomenta el aprendizaje flexible en un ambiente de pluralismo ideológico y de excelencia académica.

En el siguiente caso de la visión se omiten completamente y sobreabundan las valoraciones explícitas como nombres y calificativos:

La Universidad $\mathbf{X}$, como una de las más importantes instituciones públicas de educación superior en Colombia en cobertura, calidad y diversidad de sus servicios, aspira a consolidarse como una universidad de excelencia, pertinente, innovadora, eficiente, competitiva, con proyección internacional y de investigación científica.

Lo mismo sucede en las metas donde son menos evidentes aún:

Fortalecer y mantener actualizada la gestión institucional como apoyo permanente a la búsqueda de la excelencia académica.

Activar procesos que contribuyan a afianzar la cultura de la calidad, en la cual la descentralización, la eficiencia, la eficacia y la efectividad se constituyan en elementos dinamizadores para desarrollar planes y programas de mejoramiento continuo en búsqueda de la excelencia.

En otras palabras, la excelencia se busca en pro de la institución, a la manera de cualquier organización o empresa de índole comercial que se publicita a sí misma y a sus productos. Se evidencia una práctica discursiva en la que no se puede dejar nada sin mencionar dando la idea de completitud en tanto listas de valoraciones positivas. Según Fairclough (2003) estas funcionan como cuando se ofrece una serie de opciones en un menú donde todas aparecen atractivas a primera observación. Estas, ya sean procesos, nombres, calificativos, etc. como aposición o con el uso de la coma se constituyen en un recurso muy importante al introducir aspectos intertextuales e interdiscursivos en el texto. Estas listas texturizan una representación positiva que realiza un función de adición y 
elaboración aportando un determinado tipo de modalidad deóntica alta, y valoración implícita que imprime credibilidad al discurso. En otras palabras este tipo de estrategía del género de políticas oficiales en educación superior aparece como de alto valor de verdad donde abundarían intervenciones intertextuales que dan valor a lo que se ofrece (Fairclough, 2003) y que pone de manifiesto un rasgo ideológico de ofrecimiento en tanto bienes y servicios (Peeke, 1994; Apple, 1999) a la manera del discurso mercantíl y publicitario.

\section{Las Ciencias Sociales, las Humanidades y las Artes}

En este punto es importante destacar la casi nula referencia a las Ciencias Humanas, a las Ciencias Sociales, a la formación en Educación y a las Artes dentro de la misión, la visión y las metas de las universidades públicas. Las prácticas discursivas se han centrado en un conocimiento producido por la investigación para el desarrollo científico a la que se considera de alta calidad o de la más alta calidad dejando de lado aspectos de formación en educación, en investigación y extensión educativas que son sustanciales en el contexto de situación del país. Un importante porcentaje de los programas de pregrado en el país son licenciaturas, baste mencionar el caso de la Universidad de Nariño donde el $25 \%$ de sus programas está orientado a la formación de educadores desde cuatro diferentes facultades. No obstante, las referencias a la calidad de la educación en cuanto formación de educadores son prácticamente nulas.

La razón, representada en el discurso de la misión, la visión y las metas provendría del lado de la teoría del capital humano la cual justifica la educación solo en la medida en que esta produce un retorno productivo materialmente hablando a la inversión. Áreas de la educación superior que no representan o producen bienes y servicios (comodificación: mercantilización o comercialización de la educación) como las humanidades, las ciencias sociales, las artes, la filosofía, la literatura y particularmente los programas de licenciatura no podrían justificarse en términos de inversión (Giroux, 2001). En este sentido, las universidades públicas tendrían una representación distinta a la realidad del contexto de situación y estarían más orientadas desde sus prácticas discursivas a lo que deberían ser tanto desde la misión, como desde la visión y las metas hacia la vinculación principalmente con el sector productivo, empresarial o comercial en un énfasis profesionalizante dirigido al crecimiento económico y al razón práctica que es la crítica desde el trabajo Habermás (1992), Freire (1996) y de Chomsky (2000) a esta forma de ideología en educación superior. 


\section{Necesidades: oferta y demanda}

Buena parte de la acción de las universidades se centra en atender las necesidades de los estudiantes, de la comunidad académica y de la sociedad. Las necesidades son,

Sociales, científicas, locales/regionales/nacionales, necesidades reales de desarrollo académico, de desarrollo de la época, de la actividad educadora, etc.

Pero al concretarse se expresan las de la industria, del comercio, del sector productivo que garantizan un determinado desarrollo económico. De esta manera la universidad aparece como una institución proveedora de servicios basadas en las necesidades endógenas o exógenas que pueden ser las mismas de la institución.

Redefinir los sistemas, principios y prácticas administrativas al servicio de la academia, fomentando el desarrollo de la institución con un perfil ágil y eficiente para atender las necesidades y características de la actividad educadora.

En la anterior declaración de metas se establece la necesidad de la misma institución haciendo énfasis en estar al servicio de la academia. Se procura la agilidad y la eficiencia del accionar de la institución como en cualquier empresa dedicada a la producción de bienes y servicios. Se mitiga la fuerza de la declaración con el calificativo educadora para la actividad implícita, sin embargo, se oscurece el tipo de educación.

El recurso a las necesidades, al parecer se ha vuelto una justificación de sentido común para la ejecución de planes a corto, mediano y largo alcance sin que medie la especificidad de los problemas o de las necesidades sociales,

Crear, desarrollar, conservar, transmitir y utilizar el conocimiento para solucionar con independencia los problemas y necesidades locales, regionales y nacionales.

Aquí el término 'con independencia' podría justificar todo si no hay una explicación que satisfaga la comprensión específica de las necesidades. La lista de procesos en infinitivo es demasiado extensa para poder determinar si se acudirá a la investigación para determinar si las necesidades existen o se 
crearán las mismas para justificar la acción. Nuevamente, se aprecia una misión de la universidad como proveedora de servicios que ofrece de acuerdo con la demanda.

En las anteriores declaraciones se puede intuir la presencia de conceptos como los de oferta y demanda, implícitos en el texto. No obstante, en la siguiente aparecen explícitamente como algo de sentido común dado el uso ya cotidiano de los mismos dentro de las prácticas discursivas e interdiscursivas revelando la relación empresa-cliente; oferta-demanda; proveedor-consumidor que el texto manifiesta,

Ampliar cobertura mediante la oferta de nuevos programas/ buscando responder a las necesidades de transformación que demanda la sociedad.

Mucha de la acción que ofrecen las universidades están dirigidas a los estudiantes[15] y a la comunidad[24] (académica/universitaria/científica) como recipientes, pacientes, o beneficiarios (pasivos) de una acción directa de la institución. Las declaraciones representan a las instituciones como las que se dirigen a satisfacer las necesidades de los estudiantes o de la sociedad. No se dirigen a estos para pedirles su intervención y solicitarles su acción. La universidad pública, a través de las declaraciones de misión y visión está dispuesta o diseñada para ofrecer servicios y satisfacer necesidades. En ningún caso, por ejemplo, los estudiantes o la sociedad se comportan como sujetos (agentes) de una acción específica y directa sobre la institución, para la transformación de la misma, no obstante ser ellos el centro sobre el cual ésta gira. Las personas son en últimas los pacientes a quienes se realiza algo, como en:

\section{propiciar/formar/educar/permitir/desarrollar [en los estudiantes]}

Como en los apartados anteriores la colonización del discurso de la universidad por el del sistema político-económico del contexto global produciendose una recontextualización del discurso con características de consumismo y eficiencia, de oferta y demanda como displina del mercado que ha estado penetrando la universidad y regulando el cambio a través de prácticas discursiva en los documentos de mision, vision y metas. Esta es la razón por la que Olssen y Peters (2005) aseguran que tanto la globalización como el neoliberalismo, formas actuales de polítca económica mundial se han introducido como racionalidades estratégicas y funcionalistas para el cambio de la educación constituyéndola de esta manera como una forma de mercado. Es 
en este mismo sentido que un proceso de mercantilización como práctica discursiva asumida por la universidad pública se refleja en aspectos de la oferta y la demanda de productos y servicios (Apple, 1999; Tomasevski, 2005; Marginson, 2007; Giroux, 2001, 2009), en tanto Sosteric, Ratkovic y Gismondi (1998) consideran que este proceso ha penetrado la parte medular o estructural de la universidad.

\section{Conclusiones}

El análisis realizado al discurso de la misión, la visión y las metas de 24 universidades públicas en Colombia participantes en este estudio permite varias reflexiones a manera de conclusión.

En primer lugar, es necesario insistir que las prácticas discursivas que se desprenden de los textos de los documentos oficiales no solo reflejan una representación de la realidad, sino que constituyen una visión del mundo desde el interior de las instituciones en la que el mercado globalizante y la razón práctica son vistos como pilares fundamentales en la educación superior (Tomasevski, 2005).

De esta manera se entiende que el discurso de la misión, la visión y las metas producido por líderes o grupos administrativos de turno de las universidades evidencian un proceso de recontextualización del fenómeno educativo influenciado por la presión de políticas económicas reinantes de corte neoliberal y provenientes de un contexto global. La recontextualización del discurso de la universidad estaría dada por la sobrevaloración de aspectos provenientes de la cultura empresarial que destaca el cambio en favor de la eficiencia, la flexibilidad y la competitividad de aspectos educativos comodificados en productos, bienes y servicios, en detrimento de los actores principales que sustentan la existencia de la universidad, los estudiantes en primer lugar y los docentes e investigadores en segundo término.

La recontextualización de la misión, la visión y las metas de la universidad pública por una orientación globo-neoliberal de la educación donde el compromiso del gobierno es cada vez menor en un proceso más privatizador tendría que advertir a los defensores de la educación pública del peligro del apego al discurso empresarial, mercantil y publicitario, donde se ofrece una educación como parte de productos, bienes y servicios diseñados para grupos humanos con necesidades insatisfechas que son tratados como clientes o consumidores. La colonización del discurso educativo en la universidad desde sus documentos oficiales como orientadores de su identidad, de su acción y razón de ser por discursos traídos desde políticas y contextos exógenos que 
promueven la razón práctica del mercado subvalora la educación que 'no produce' materialmente o no entrega productos o evidencias tangibles de los mismos.

Esta es la razón por la que movimientos estudiantiles han luchado y siguen enarbolando como argumento: la no privatización de la educación pública. En realidad, es el discurso que ha estado cambiando desde el advenimiento del neoliberalismo como fenómeno político y económico catalizado por el fenómeno de la globalización e impulsado por el desarrollo de las nuevas tecnologías de la información en un contexto que hace posible su penetración a nivel masivo (Giroux, 2009). Pero ese cambio discursivo también, como se ha podido ver en este estudio, ha penetrado el discurso en educación superior a través de prácticas discursivas en los documentos sobre la misión, la visión y las metas de la universidad pública dando lugar a un proceso de recontextualización ideológica que afecta sus representaciones de la realidad educativa, de sus imaginarios colectivos, y la misma conceptualización de la educación y la universidad pública. La representación de la universidad pública desde la misión, la visión y las metas es prácticamente la de la institución privada que privilegia la razón práctica y los valores en términos de producción de bienes y servicios para la sociedad que busca solución a sus necesidades de formación en una relación de tipo empresarial donde impera la ley de la oferta y la demanda.

El cambio en el discurso es el primer paso para la acción en la toma de decisiones por el cambio de la realidad educativa: lo que se piensa y lo que se dice discursivamente afectará directamente lo que se hace. La universidad como institución pública ha estado representando y transmitiendo a través de prácticas discursivas afectadas por del nuevo contexto político-económico durante las primeras décadas del siglo XXI un discurso recontextualizado de la misión, visión y metas. Consecuentemente, cuando se habla de la privatización de la universidad se entiende la representación discursiva de la universidad en términos del discurso ideológicamente recontextualizado en la privatización. Cada vez más se piensa en una universidad con las características del tipo privado donde lo que cuenta es el lucro (Chomsky, 2000) y donde los intereses privados de corte neoliberal, el énfasis en la formación de profesionales de alta calidad para el mercado laboral, para la industria y el crecimiento económico han sido centrales. Lo anterior enfatiza las valoraciones positivas de la alta calidad, de la eficiencia, de la flexibilidad y de la competitividad económicas aplicadas ya a los currículos, y a las instituciones públicas como también a la gente para asemejarlas a lo privado como sinónimo de eficiente prevaleciendo 
un proceso diacrónico de una cada vez mayor desvinculación financiera del estado en el énfasis técnicamente profesionalizante de las instituciones educativas a expensas de las ciencias humanas, sociales, artísticas y creativas de formación educativa y socio-crítica.

Finalmente, el proceso de recontextualización como reorientación del discurso de la universidad pública determinado en este estudio está históricamente configurado y ha sido mucho más evidente en un contexto como el de finales de la década del siglo XX en el cual se han ido venciendo las barreras espacio-temporales para el acceso a la información y al conocimiento, pero al mismo tiempo se han abierto las puertas a nuevas formas de intervención, aunque ya no de manera coercitiva como en el pasado pero si de forma ideológica a través del discurso. Una vez que el mapa político y económico ha quedado sin barreras (globalización neoliberal), se ha dado paso para que la lucha por la hegemonía de los sistemas dominantes se lleve a cabo con la nueva configuración de un nuevo capitalismo (Boltanski y Chiapello, 2002), a través de nuevas prácticas y tecnologías discursivas que han servido para acelerar este proceso de cambio en una universidad sin barreras pero al mismo tiempo con predominio discursivo de tipo económico y global.

\section{Nota}

${ }^{1}$ Artículo producto del proyecto de investigación del mismo nombre aprobado y financiado por el Sistema de Investigaciones de la Universidad de Nariño.

\section{Referencias}

Apple, M. (1996). Política cultural y educación. Madrid: Morata.

Apple, M. (1999). Freire, Neo-liberalism and education. Discourse: studies in the cultural politics of education, 20(1), 5 .

Aronowitz, S. (2001). The last good job in America: work and education in the new global technoculture. Lanham, Md.: Rowman \& Littlefield : Distributed by National Book Network.

Boltanski, L., \& Chiapello, E. (2002). El nuеvo espíritu del capitalismo. Madrid: Ediciones Akal, S. A.

Brown, J. K. (1984). Corporate Soul Searching: The Power of Mission Statements. Across the Board, 44-52.

Castells, M. (1998). La era de la información: economía, sociedad y cultura. Vol. 1, La sociedad red. Madrid: Alianza Editorial.

Connell, I., \& Galasinski, D. (1998). Academic Mission Statements: An Exercise in Negotiation. Discourse \& Society, 9(4), 457-479. 
Consejo Nacional de Acreditación. (2014). Lineamientos para la Acreditación Institucional. Santafé de Bogotá D.C. CNA.

Chomsky, N. (2000). El beneficio es lo que cuenta: neoliberalismo y orden global. Barcelona: Crítica.

Chomsky, N., \& Dieterich, H. (2002). La sociedad global: educación, mercado y democracia. Tlalnepantla, Mexico: Contrapuntos.

Drucker, P. F. (1974). Management: tasks, responsibilities, practices. New York,: Harper \& Row.

Fairclough, N. (1989). Language and power. London: Longman.

Fairclough, N. (1992). Discourse and social change. Cambridge, UK: Polity Press.

Fairclough, N. (1993). Critical discourse analysis and the marketization of public discourse: the universities. Discourse \& Society, 4(2), 133-168.

Fairclough, N. (1995). Critical discourse analysis: the critical study of language. London: Longman.

Fairclough, N. (2003). Analysing discourse: textual analysis for social research. London: Routledge.

Fowler, R., Hodge, B., Kress, G., \& Trew, T. (1979). Language and control. London: Routledge \& Kegan Paul.

Fox, J. (2004). Chomsky y la globalización. Barcelona: Editorial Gedisa S.A.

Freire, P. (1996). Cartas a Cristina: reflexiones sobre mi vida y mi trabajo. México: Siglo XXI.

Giddens, A. (2000). Un mundo desbocado: los efectos de la globalización en nuestras vidas. Madrid: Taurus.

Giroux, H. (2001). Vocationalizing higher education: Schooling and politics of corporate culture. In H. Giroux \& K. Myrsiades (Eds.), Beryond the corporate university: Culture and pedagogy in the new millenium. New York: Rowman \& Littlefield.

Giroux, H. (2009). Neoliberalism, Corporate culture, and the promise of Higher Education: The University a Democratic Public Sphere. Harvard Educational Review, 72(4), 425-464.

Graham, P. (2001). Space: Irrealis Objects in Technology Policy and their Role in a New Political Economy. Discourse \& Society, 12(6), 761-788.

Grant, D. (Ed.). (2005). Discourse and Organizational Change: Emerald Group Publishing Limited.

Habermas, J., \& Blazek, J. (1987). The idea of the University: Learning Processes. New German Critique(41), 3-22.

Habermas, J. (1992). Ciencia y técnica como "ideología". Madrid: Tecnos. 
Halliday, M. (1982). El lenguaje como semiótica social. México: Fondo de Cultura Económica.

Halliday, M. \& Martin, J. (1993). Writing science: literacy and discursive power. Pittsburgh: University of Pittsburgh Press.

Jenkins, S. (1995). Accountable to none: the Tory nationalization of Britain. London: Hamish Hamilton.

Kanter, R. M. (2002). Evolve!: para triunfar en la cultura digital del mañana. Bilbao: Ediciones Deusto, S. A.

Kress, G., \& Hodge, B. (1979). Language as ideology. London: Routledge \& Kegan Paul.

Marginson, S. (2007). University Mission and Identity for a post Post-public era. Higher Education Research \& Development, 26(1), 117-131.

Martín-Barbero, J. (2000). Una mirada latinoamericana a la sociedad de la información. En UNICOM (Ed.), Desafíos de la sociedad de la información en América Latina y Europa (pp. 27-33). Santiago de Chile: Editorial LOM.

Meyer, M. (2003). Entre la teoría, el método y la política; la ubicación de los enfoques relacionados con el ACD. En R. Wodak \& M. Meyer (Eds.), Métodos de análisis crítico del discurso (pp. 35-59). Barcelona: Gedisa.

Olssen, M., \& Peters, M. (2005). Neoliberalism, higher education and the knowledge economy: from the free market to knowledge capitalism. Journal of Education Policy, 20(3), 313-345.

Peeke, G. (1994). Mission and Change: Institutional Mission and its Application to the Management of Further and Higher Education. Buckingham England; Bristol, PA: Society for Research into Higher Education \& Open University Press.

Poster, M. (1990). The mode of information: poststructuralism and social context. Chicago: University of Chicago Press.

Reboul, O. (1986). Lenguaje e ideología. México, D.F.: Fondo de Cultura Económica.

Sádaba Rodríguez, I. (2002). La conflictividad en la sociedad de la información y la globalización: de la 'cuestión social' al discurso del riesgo. Nómadas, (5), 26.

Santana-Seda, O. (1975). A constrastive study in rhetoric: an analysis of the organization of English and Spanish paragraphs wirtten by native speakers of each language. New York University.

Scott, P. (Ed.). (2000). Higher education reformed. London; New York: Falmer Press. 
Sosteric, M., Ratkovic, G., \& Gismondi, M. (1998). The university, accountability, and market discipline in the late 1990s. Electronic Journal of Sociology, 3(3).

Tomasevski, K. (2005). Globalizing What: Education as a Human Right or as a Traded Service? Indiana Journal of Global Legal Studies, 12(1), 1-78.

Van Dijk, T. A. (1998). Ideología: un enfoque multidisciplinario. Barcelona: Editorial Gedisa, S.A.

Wendt, R. (1994). Learning to 'walk the talk': a critical tale of the micropolitics at a total quality university. Management Communication Quarterly, 8(1), 5-45.

Wodak, R. (2003). De qué trata el análisis crítico del discurso (ACD)? En R. Wodak \& M. Meyer (Eds.), Métodos de análisis crítico del discurso (pp. 17-34). Barcelona: Gedisa.

Wodak, R., \& Fairclough, N. (2010). Recontextualizing European higher education policies: the cases of Austria and Romania. Critical discourse Studies, 7(1), 19-40.

Yang, R. (2003). Globalisation and higher education development: a critical analysis. International Review of Education, 49(3/4), 269-291.

\section{Nota biográfica}

\begin{tabular}{|l|l|}
\hline Jorge E. Benavides B. es doctor en Ciencias de la Educación, \\
Universidad de Nariño-RUDECOLOMBIA. Magíster en \\
Lingüística Aplicada para TEFL, Ohio University, Athens, \\
USA. Especialista en Traducción Inglés-español, Universidad \\
de Nariño. Docente investigador Titular adscrito al \\
Departamento de Lingüística e Idiomas, Facultad de Ciencias \\
Humanas, Universidad de Nariño (Colombia). Director del \\
Grupo Interdisciplinario de investigación TICED \\
(Categorizado en COLCIENCIAS). Áreas de investigación: \\
Análisis del Discurso, Uso pedagógico de las NTIC, Medición \\
del nivel de inglés como lengua extranjera, Comprensión de \\
lectura. E-mail: jbenavides@ @udenar.edu.co
\end{tabular}

